\title{
Fields and coupling between coils embedded in conductive environments
}

\author{
Son $\mathrm{Chu}^{*}$, Andrea Vallecchi, Christopher J. Stevens, and Ekaterina Shamonina \\ Department of Engineering Science, University of Oxford, Oxford OX1 3PJ, UK
}

Received: 15 September 2017 / Accepted: 17 November 2017

\begin{abstract}
An approximate solution is developed for the mutual inductance of two circular coils enclosed by insulating cavities in a conducting medium. This solution is used to investigate the variation of the mutual inductance upon the conductivity of the background (e.g., soil, seawater or human body), as well as upon other parameters such as the vertical of the coils and the displacement of one of the coils in the horizontal plane. Our theoretical results are compared with full wave simulations and a previous solution valid when a conductive slab is inserted between two coupled resonant coils. The proposed approach can have direct impact on the design and optimisation of magnetoinductive waveguides and wireless power transfer for underground/underwater networks and embedded biomedical systems.
\end{abstract}

Keywords: Coils / conducting materials / mutual inductance / magnetic fields / eddy currents / magnetoinductive waves / wireless underground / underwater communications networks

\section{Introduction}

Faraday's law of induction describes how a time-varying magnetic field introduces an electromotive force in any nearby electric circuit. It is the fundamental principle of the operation of transformers, motors and generators. Recently, the use of magnetic induction for communications has become increasingly popular in RF-challenging environments, e.g., underground/underwater or in vivo medical diagnosis and treatment applications [1-3]. These media are moderately conductive and represent a challenge to conventional wireless links using transverse electromagnetic (TEM) waves. Their main disadvantages are high ohmic losses, a rapid increase of the attenuation with frequency and the need for large antennas [4]. In order to achieve longer range and more reliable communications, several authors considered the use of magnetoinductive (MI) waveguides [1-3].

MI waveguides supporting propagation of MI waves were first proposed in $[5,6]$ and there is by now a significant literature on the subject [7]. Basically, an MI waveguide is a one, two or three dimensional array of resonant magnetic circuits (elements) that support the propagation of waves by virtue of their mutual magnetic coupling. In fact, MI waves have some advantages over TEM wave-based techniques used for terrestrial wireless communications in that they do not require large antennas, are less affected

\footnotetext{
* e-mail: son.chu@eng.ox.ac.uk
}

by the variability of the underground and underwater channel conditions, and are independent from other environment impairments such as multipath fading [1,2]. Also, since the MI waveguides are constructed from isolated discrete resonators, they can be quite flexible and resilient compared to a continuous traditional waveguide. While most materials in nature are nonmagnetic, they are often conductive [8,9], resulting in an attenuation of the near fields which enable the coupling between resonators. When an MI waveguide is embedded in a conductive medium, eddy currents generated by the coupling magnetic flux are, in general, expected to lower the inter-element coupling strength, which will in turn reduce the MI waveguide bandwidth and increase their attenuation. This makes the behaviour of coupled resonant coils inside a conductive medium an important subject of investigation for communications in dissipative media.

Apart from wireless underground/underwater communications networks (WUCNs), the study of coupled resonant coils immersed in a dissipative medium is of great interest in embedded biomedical systems and in body area networks for health-related applications, since the human body and other biological tissues are moderately conductive at microwave frequencies within the range of conductivity from about $0.05 \mathrm{~S} / \mathrm{m}$ (fat) to about $1.5 \mathrm{~S} / \mathrm{m}$ (blood) [9].

In the last century, there has been some interests in the subject of radiating systems embedded in conductive media due to the need of submarine communications [10-14]. The uninsulated Hertzian dipole in an infinite homogenous 




(a)



(b)

Fig. 1. (a) Persepctive and (b) cross section of the investigated configuration consisting of two coils with the same orientation, completely immersed in a conductive medium. Each coil is insulated by a dielectric box to prevent current leakage.

conductive medium was first investigated in [10]. It has been concluded there that a detectable field strength at some distant points from the source can only generated by a massive power due to extremely large ohmic losses in the immediate neighbourhood of the dipole. The dipole therefore must be insulated in order to control current distribution and radiation. Later, it was shown in [11] that the efficiency of a magnetic dipole is higher than that of its electrical counterpart in conductive environments. However, all these works have assumed a simple point dipole source concentrating on far-field propagation and usually ignoring the reactive near fields.

In the intensive theoretical and experimental works for an insulated electric dipole in conductive media by King [15-18], it is stated that: The near electric field of a bare or insulated dipole in a medium like muscle or the earth is much more involved than the far field because it is elliptically polarized. Due to the symmetry of electromagnetic fields, one might expect the magnetic field of an insulated loop current inside dissipative media to be elliptically polarized as well. However, to the best of the authors knowledge, there was no published work studying the impact of the conductive surrounding environments on the polarization of the fields, especially the magnetic field, generated by a loop current before recent studies $[19,20]$.

In these works $[19,20]$, it has been confirmed by numerical simulations and experiments that the presence of an intervening conducting block between two coils has two major effects on the magnetic field: firstly, it causes the polarisation of the magnetic field to become elliptical, and secondly, it introduces a phase shift in the magnetic field that leads to a complex mutual inductance. However, the configuration considered in $[19,20]$ was relevant to the case when a conductive slab is inserted between two coupled resonant coils. In this paper we focus instead on the case when a MI waveguide is completely immersed in a conductive medium, e.g. buried in the ground as in WUCNs. The basic unit of this configuration consists of two identical coils with the same orientation placed inside insulating dielectric boxes in an infinite homogeneous dissipative environment, as illustrated in Figure 1.

The aim of the present work is to develop an analytical method to more accurately estimate the fields, and thereby the mutual inductance of these two low frequency coils coupled in close proximity inside a conducting medium. In particular, our main interest is the behaviour of the mutual inductance as a function of medium conductivity, vertical separation between coils, as well as the displacement of the receiver from the transmitter axis. The analysis follows the approach used by Dodd and Deeds (D\&D) in [21], where the differential equation for the magnetic vector potential is derived and solved subject to the boundary conditions valid for infinitely wide conductive layers. Another fundamental assumption in this paper is that at low frequencies, the displacement current is negligible compared to the conduction current. The expressions of the field will be given in terms of integrals of Bessel functions. Once the magnetic vector potential is known, every other physically observable electromagnetic quantity can be calculated, including the mutual inductance of the coils.

The rest of this paper is organized as follows. In Section 2, we formulate the problem and develop a new formula which is referred to as 'multilayer solution' for the magnetic vector potential of an insulated coil immersed in conductive environments. The full expression of the field is provided in the Appendix. Then, in Section 3 the analytical estimation obtained with the multilayer solution are compared with D\&D and full wave simulation results for variable conductivity of the background medium, distance between coils, and horizontal displacement of the transmitter and receiver. To gain insight in the results for the mutual inductance, in Section 4 the magnetic field distribution for the configuration in Figure 1 is discussed. Finally, the concluding remarks are drawn in Section 5.

\section{Analytical solution}

The cross section of the configuration under analysis is shown in Figure $1 \mathrm{~b}$ two identical coils of radius $r_{0}$ are placed at a vertical distance $d$ from each other inside a conducting medium of conductivity $\sigma$. To prevent current leakage, the coils need to be insulated from the surrounding environment by putting them into small dielectric boxes. These boxes having no particular influence on the magnetic channels are assumed to be free space.

To derive the analytical solution for the magnetic vector potential, the dielectric boxes are replaced by infinitely wide dielectric layers, as shown in Figure 2. Space is divided into the six sections labelled with Roman numbers in Figure 2. The transmitting coils is driven by a 
current density $J_{0}$. The receiving coil is left open circuited. For most coil problems at low frequency, we can rely on their quasi-static solution where the current density is assumed to be uniform along the coils. Then, the configuration of the field produced by the transmitting coil will have axial symmetry. With these assumptions, the current density has only one spatial component along the azimuthal angle $\theta$ and the same applies to the magnetic vector potential $\mathbf{A}\left(0, A_{\theta}, 0\right)$. Since the size of the coil cross section has little influence on the results, the coils are assumed to be filamentary. Hence, the total driving current in the transmitting coil is taken as:

$$
I=\frac{J_{0}}{\delta\left(\rho-r_{0}\right) \delta\left(z-z_{0}\right)},
$$

where $\rho$ and $z$ is the cylindrical coordinates, $r_{0}$ and $z_{0}$ is the cylindrical coordinates of the transmitting coil, $\delta(a)$ is the delta function of variable $a$.

All the media are assumed to be linear, isotropic and homogeneous. Since the displacement is neglected at low frequencies $\left(\omega \varepsilon_{0} \varepsilon_{r} \ll \sigma\right)$, the general differential equation of the magnetic vector potential can be written [21]:

$$
\begin{aligned}
& \frac{\partial^{2} A_{\theta}^{(n)}}{\partial \rho^{2}}+\frac{1}{\rho} \frac{\partial A_{\theta}^{(n)}}{\partial \rho}+\frac{\partial^{2} A_{\theta}^{(n)}}{\partial z^{2}}-\frac{A_{\theta}^{(n)}}{\rho^{2}}-j \omega \mu_{0} \sigma_{n} A_{\theta}^{(n)} \\
& \quad=-\mu_{0} \mathrm{I} \delta\left(\rho-r_{0}\right) \delta\left(\mathrm{z}-z_{0}\right)
\end{aligned}
$$

where $A_{\theta}^{(n)}$ is the vector potential in the $n$-th region, which only depends on the cylindrical coordinates $\rho$ and $z$ due to the symmetry assumption, $\omega=2 \pi f$ is the angular frequency, $f$ is the working frequency, $\varepsilon_{0}$ is the permeability of free space, $\varepsilon_{r}$ is the relative permeability of materials, $\mu_{0}$ is the permeability of free space, $\sigma_{n}$ is the conductivity of the $n$-th region.

By using the mathematical method adopted in [21] to solve (2), the magnetic vector potential in each region is expressed in the general form:

$$
A_{\theta}^{(n)}(\rho, z)=\int_{0}^{\infty}\left(A_{n} e^{-\alpha_{n} z}+B_{n} e^{\alpha_{n} z}\right) J_{1}(\alpha \rho) d \alpha,
$$

where $A_{n}$ and $B_{n}$ are functions of the separation constant $\alpha, \alpha_{n}=\sqrt{\alpha+j \omega \mu_{0} \sigma_{n}}$ and $J_{1}$ is the Bessel function of the first kind. In particular, $B_{\mathbf{I}}=0$ and $A_{\mathbf{V} I}=0$ to ensure the vanishing of the vector magnetic potential in regions $(\mathbf{I})$ and (VI) when $z$ goes to plus and minus infinity, respectively, and $A_{\mathbf{I}}, A_{\mathbf{I} I}, B_{\mathbf{I} I}, \ldots, A_{\mathbf{V}}, B_{\mathbf{V}}$ and $B_{\mathbf{V} I}$ are solutions of the system of 10 linear equations (7) in the appendix which derive from the continuity conditions for the electric and magnetic field components at layer interfaces. By solving the system of linear equations (7) and substituting its solutions into (3), an analytical solution for the magnetic vector potential over all space regions is obtained (see Appendix). Once the magnetic vector potential is known, the induced voltage in the receiving coil may be obtained as:

$$
V=j \omega \int A \cdot d S
$$

Recalling that the Cartesian coordinates of the points along the circumference of the receiving coil are $(x$, $y)=\left(x_{0}+r_{0} \cos \phi, y_{0}+r_{0} \sin \phi\right)$, where $\left(x_{0}, y_{0}\right)$ is the centre

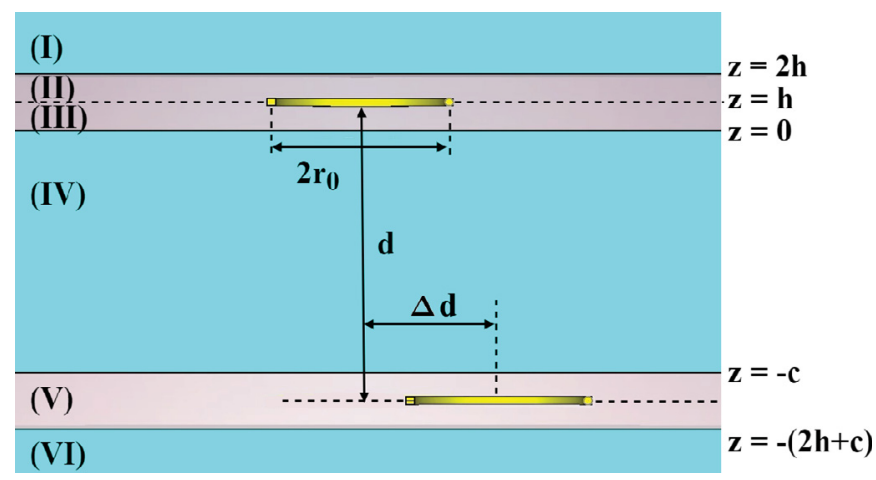

Fig. 2. Theoretical model of two insulated coils in a conductive medium where the finite size dielectric enclosures for coil insulation of the actual configuration are considered as infinite dielectric layers.

point of the receiving coil, and $\phi$ is a local angular coordinate varying from 0 to $2 \pi$. The induced voltage can be rewritten as:

$$
V=j \omega \int_{0}^{2 \pi} r_{0} \cos (\theta-\varphi) A_{\theta}^{(\mathbf{V})}(\rho, z) d \varphi,
$$

where $\rho=\sqrt{\left(x_{0}+r_{0} \cos \phi\right)^{2}+\left(y_{0}+r_{0} \sin \phi\right)^{2}}$, and $\theta=\operatorname{arc}-$ $\tan \left[\left(y_{0}+r_{0} \sin \phi /\left(x_{0}+r_{0} \cos \phi\right)\right)\right]$. Finally, the mutual inductance between two coils is determined as the ratio of the induced voltage in the receiver over the total current in the transmitter:

$$
M=\frac{V}{j \omega I} .
$$

\section{Mutual inductance}

The developed analytical solution is validated by comparing the theoretical predictions of the mutual inductance of the two coil system in Figure 2 with those obtained by full wave simulations with CST microwave studio (CST MWS) [22] of the original configuration in Figure 1 and the D\&D solution in [21]. Firstly, we examine how the magnetic coupling varies as a function of the medium conductivity for the case of two coaxial coils. The analytical results calculated with our multilayer solution are compared with D\&D results [21] and simulation results corresponding to three different sizes of the finite cylindrical free space regions providing coil insulation (cf. Fig. 1), whose radius $R$ is set to be $R=1.1 r_{0}, 2 r_{0}$, and $3 r_{0}$. The coils are enclosed into insulating dielectric boxes with a fixed height of $2 h=7 \mathrm{~mm}$. Figure 3 analyses the impact of the conductivity of the surrounding medium on the modulus and phase of the mutual inductance.

As apparent from Figure 3, the multilayer solution is in good agreement with the CST full wave simulations, whereas D\&D solution is less accurate. In fact, when the conductivity of the medium is small enough, the field produced by the transmitting coil expands through the intermediate conducting slab. Eddy currents are excited both inside the upper and the lower conducting half-spaces 


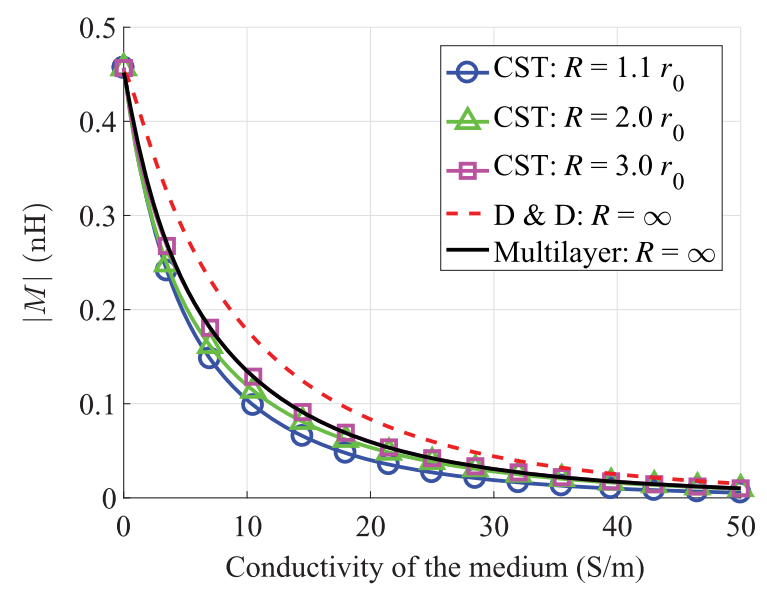

(a)

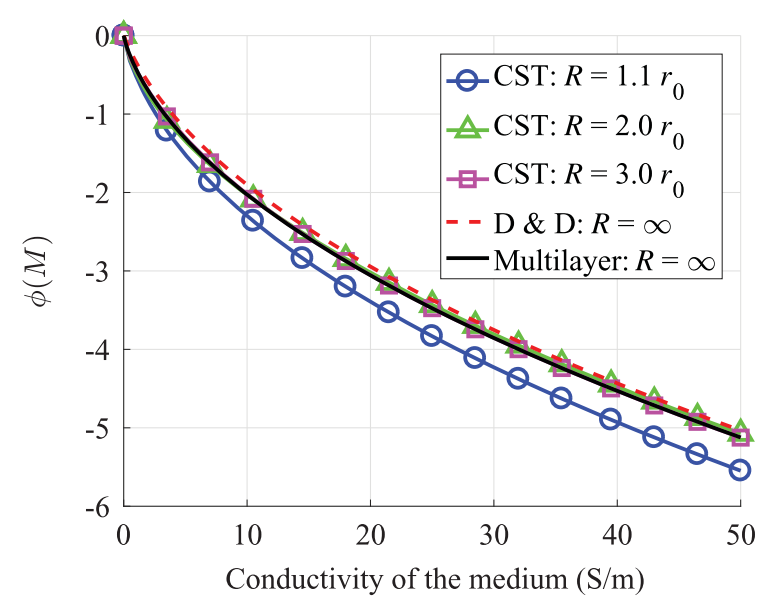

(b)

Fig. 3. Mutual inductance $(M)$ of two coaxial circular coils of radius $r_{0}=11 \mathrm{~mm}$ and spaced apart $d=37 \mathrm{~mm}$ inside a conductive medium with conductivity variable from $\sigma=0$ to $\sigma=50 \mathrm{~S} / \mathrm{m}$. (a) Modulus and (b) phase of $M$ obtained by the multilayer solution are compared with D\&D solutions [21] and CST MWS simulation results for three different sizes of the insulating boxes.

and their fields that contribute to the total magnetic field threaded with the receiving coil. Once the conductivity grows sufficiently large, the upper and lower media become more impermeable and progressively their contribution to the total magnetic field in the vicinity of the receiver declines. The new analytical solution then coincides with the original D\&D. However, the range of conductivity of media from 0 to $10 \mathrm{~S} / \mathrm{m}$ is critical for $\mathrm{MI}$ communication applications because the conductivities of human tissues and organs, water, sea water and most soil types fall in that range $[8,9]$.

Furthermore, we have compared the relative errors of the D\&D and multilayer solutions with respect to CST simulation, as shown in Figure 4 . When the radius of the dielectric box is three times larger than the coil radius $R \geq 3 r_{0}$, the multilayer solution is found to be in excellent agreement with CST simulations in terms of modulus of the mutual inductance with an average relative error less than $2 \%$ over the range of conductivity from $\sigma=0$ to $\sigma=50 \mathrm{~S} / \mathrm{m}$.

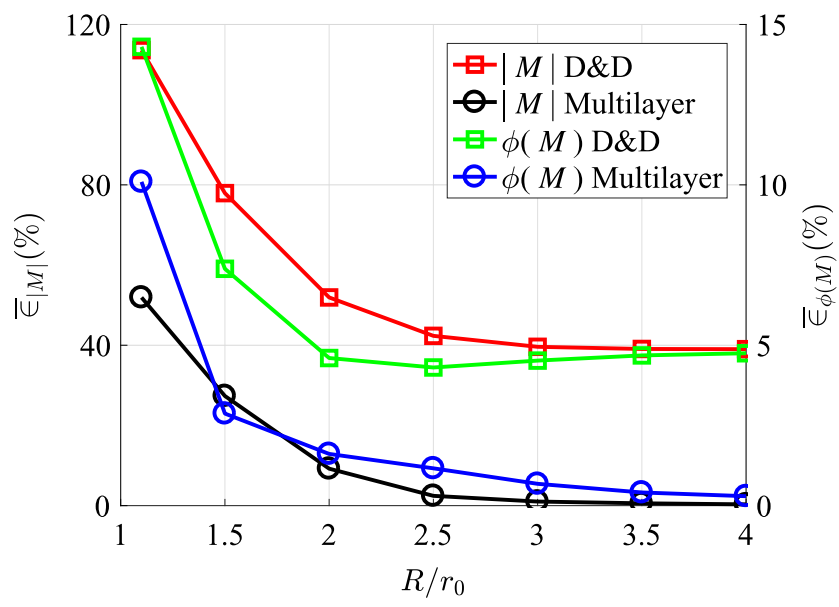

Fig. 4. Average relative errors of the $D \& D$ and the multilayer solutions in terms of modulus and phase of the mutual inductance with respect to CST simulation results for several different sizes of the insulating boxes.

On the other hand, the D\&D solution seems unable to accurately describe the magnetic coupling magnitude and is associated with an average relative error with respect to simulated data being larger than at least $38 \%$ for all cases. Additionally, while both of them exhibit satisfactory accuracy in the estimation of the phase of the mutual inductance, the multilayer solution is noticeably more accurate.

Next, the effects of the distance between the coils are investigated. Since the height of the dielectric boxes is kept constant, the vertical separation between the coils is actually related to the thickness $c$ of the conductive layer (IV). Figure 5 shows the mutual inductance as a function of the normalised vertical separation between the transmitting and receiving coils when the conductivity of the medium is set to $\sigma=10 \mathrm{~S} / \mathrm{m}$. As in previous Figure 3, a set of three numerical simulations corresponding to three different dielectric box radii is examined: $R=1.1 r_{0}, 2 r_{0}$, and $3 r_{0}$. Again, the results look similar to those in Figure 3 where the novel analytical solution appears to constitute a better approximation. The magnitude of the magnetic coupling may be seen to decline as the separation between the coils increase. Indeed, the magnetic field decays quickly for increasing separation following an inverse cube law. Moreover, an increase in distance means a thicker conductive layer between the coils which leads to stronger eddy currents. The induced field therefore reduces the primary field generated by the transmitting coil and accordingly lowers the mutual inductance. The rule of thumb $R \geq 3 r_{0}$ is still valid, and when the radius of the insulator is sufficiently large, the multilayer formula gives an approximate value of the magnetic coupling, which is accurate to better than a $2 \%$ relative error in comparison to simulation results.

Finally, we have found an interesting behaviour of the mutual inductance when the circular symmetry is broken. The conductivity of the medium is still fixed at $\sigma=10 \mathrm{~S} / \mathrm{m}$ but now the receiving coil is displaced along the $y$-axis by a distance $\Delta d$ away from the coaxial alignment. In free space, or in other words, when the conductivity of the 




(a)

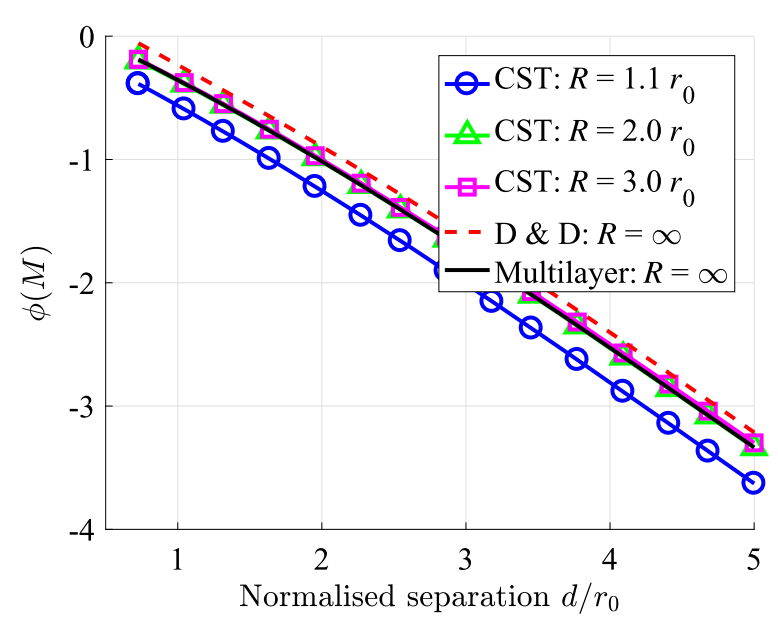

(b)

Fig. 5. Mutual inductance $(M)$ of two coaxial circular coils of radius of $r_{0}=11 \mathrm{~mm}$ inside a conductive medium with conductivity of $\sigma=10 \mathrm{~S} / \mathrm{m}$ as a function of normalised vertical separation $d / r_{0}$. (a) Modulus and (b) phase of $M$ obtained by the multilayer solution are compared with D\&D solutions [21] and CST MWS simulation results for three different sizes of the insulating boxes.

medium is zero, the magnetic field generated by a uniform circular loop current is symmetric with the strongest magnetic flux density along the axis of the loop. When the receiver moves off the coaxial configuration, the larger the displacement from the receiving loop centre to the transmitter axis is, the more the modulus of the mutual inductance declines until it reaches the zero-point where the net magnetic field flux across the loop vanishes. Then its modulus starts increasing due to the negative coupling. For the considered coil configuration in the air, the zeropoint occurs at about $\Delta d / r_{0}=5$. However, in the presence of a surrounding dissipative medium, the modulus of the mutual inductance does not monotonically decrease until reaching the zero-point as the receiving coil is moved further apart from the transmitting coil, as shown in Figure 6, where the modulus and phase of the mutual inductance are presented as a function of the horizontal displacement of the receiver.

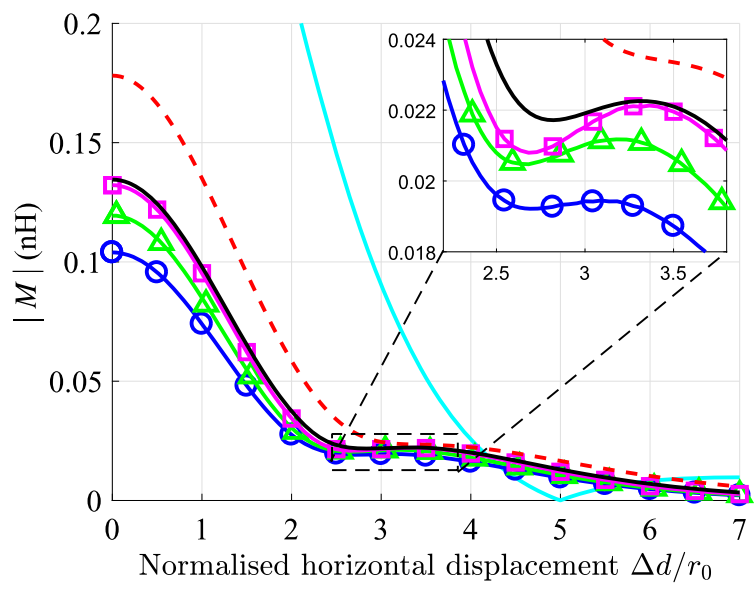

(a)



(b)

Fig. 6. Mutual inductance $(M)$ of two circular coils of radius of $r_{0}=11 \mathrm{~mm}$ and spaced apart $d=37 \mathrm{~mm}$ inside a conductive medium with conductivity of $\sigma=10 \mathrm{~S} / \mathrm{m}$ as a function of the normalised horizontal displacement $\Delta d / r_{0}$. (a) Modulus and (b) phase of $M$ obtained by the multilayer solution are compared with D\&D solutions [21] and CST MWS simulation results for three different sizes of the insulating boxes. The mutual inductance of two coils in free space (air) is also inlcuded.

The multilayer solution once again shows a higher accuracy than the D\&D formula. At a large distance from the transmitting coil axis, the $\mathrm{D} \& \mathrm{D}$ formula may give satisfactory result for the magnitude of the mutual inductance but not for its phase. Furthermore, it generally fails to provide a good approximation of the mutual inductance as a function of the receiver horizontal displacement, which is instead adequately reproduced by the new multilayer formula. In contrast to the free space case, the magnetic coupling is not a monotonically decreasing function of the displacement before the zeropoint. In fact, there is no longer a zero-point when coils are embedded in a dissipative material. Also, the mutual coupling may have both local minimum and maximum values over a certain range of the horizontal displacement, as can be seen in Figure 6 . The reason underlying this trend will be further clarified in the next section by examining the distribution of the magnetic field. 


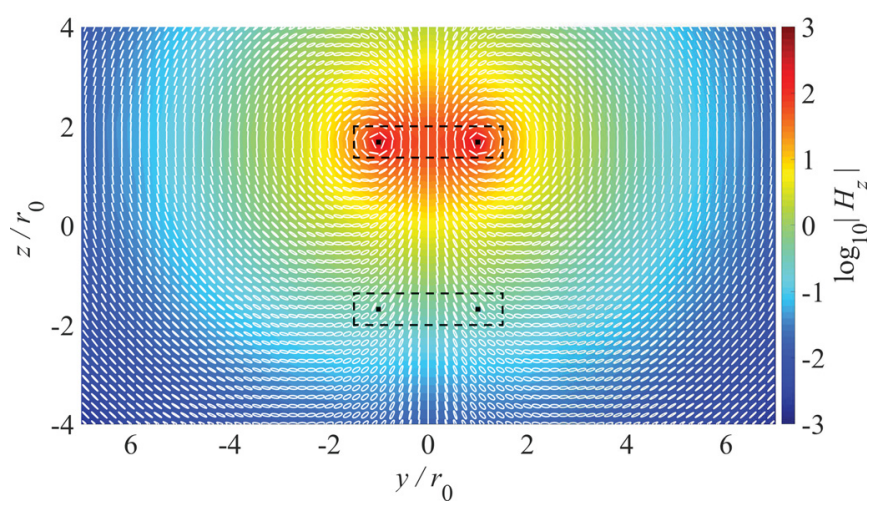

Fig. 7. Polarisation ellipses and magnitude of the magnetic field produced by a transmitter coil of radius of $r_{0}=11 \mathrm{~mm}$ when a coaxial receiver coil displaced by $37 \mathrm{~mm}$, and two coils are insulated by two cylindrical dielectric boxes with the height $2 h=7 \mathrm{~mm}$. Ellipse axes are normalised and plotted at a sampling step of $2 \mathrm{~mm}$ along the $\mathrm{y}$ and $\mathrm{z}$ directions. Black dash lines mark the cross sections of the dielectric boxes. The conductivity of the block is $\sigma=10 \mathrm{~S} / \mathrm{m}$. The mutual inductances is $M=0.11 \angle-122^{\circ}$.

\section{Magnetic field}

Figure 7 shows the results of a finite element calculation by CST [22] for the magnitude and polarisation of the magnetic field when the insulated transmitting and receiving coils are immersed in a dissipative medium in a coaxial disposition.

Obviously, the magnetic field generated by the transmitting coil in the air is linearly polarised. However, due to the impact of eddy currents in the background medium and their associated field, the total magnetic field turns out to be elliptically polarised, i.e. its spatial components have a nonzero phase shift relative to one another. Correspondingly, the mutual inductance between the transmitting and receiving coils will become a complex quantity.

As it can be seen from Figure 7, in the plane of the receiver, the size and tilt angle of the ellipses representing the polarization of the magnetic field change noticeably between $\Delta d=2.5 r_{0}$ and $\Delta d=3.5 r_{0}$. It means that the $H_{z}$ component, which determines the value of the induced voltage at the receiver changes in modulus as well as in its relative phase with respect to the $H_{\rho}$ component. The distribution of the $z$-component of the magnetic is further explored in the contour plots of Figure 8 both when the coils are in free space and immersed in a conductive medium with $\sigma=10 \mathrm{~S} / \mathrm{m}$.

These maps are expectedly consistent with the analytical prediction of the mutual inductance by the multilayer formula and corresponding simulation results shown in Figure 6 . When the two coils are in free space, cf. Figure 8a, a region where the field vanishes appears at about $\Delta d=5 r_{0}$, similarly to Figure 6a. Otherwise, when the two coils are immersed in a dissipative environment, eddy currents are excited inside the medium. Due to the interference between the primary field created by the transmitting coil and the field produced by eddy currents, which can be substantially phase shifted with respect to the inducing field, the contour map of the magnitude of the $H_{z}$ component no longer exhibits

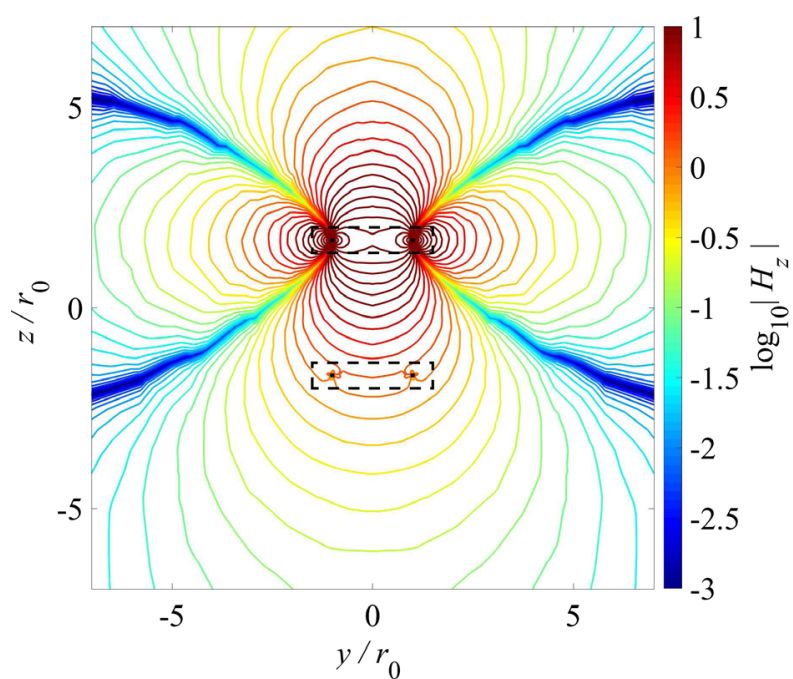

(a)

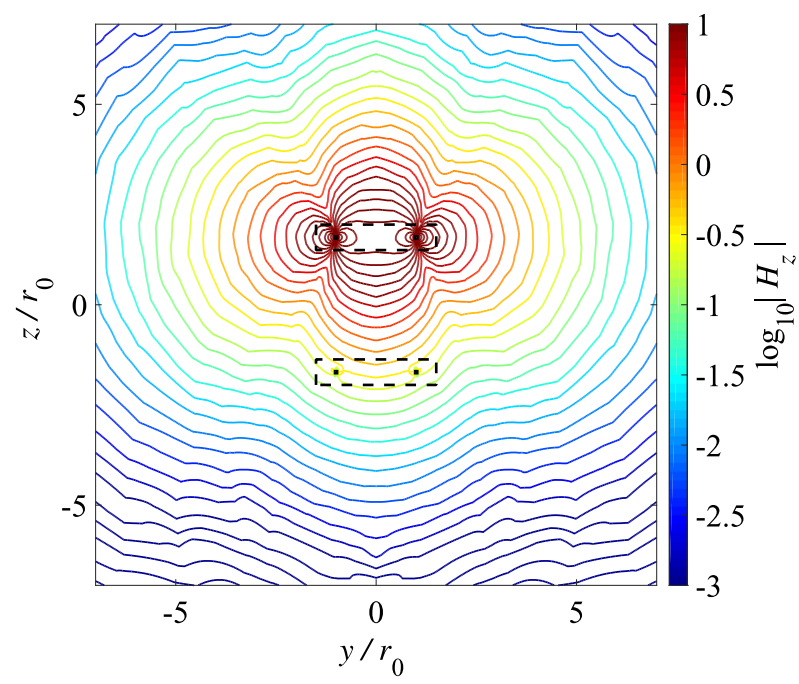

(b)

Fig. 8. Contour map of the modulus of $H_{z}$ component of the magnetic field generated by a transmitting coil with a radius of $r_{0}=11 \mathrm{~mm}$ and spaced apart $d=37 \mathrm{~mm}$ inside a conductive medium with conductivity (a) $\sigma=0$ and (b) $\sigma=10 \mathrm{~S} / \mathrm{m}$. Black dash lines mark the cross sections of the insulating dielectric boxes.

null regions where the net flux of the magnetic field vanishes but has instead a smoother quatrefoil distribution, as shown in Figure 8b. Since the mutual inductance is calculated by integrating the $H_{z}$ component across the area of the receiver, the quatrefoil geometry of the $H_{z}$ component results in the local minimum and maximum points of the mutual inductance as a function of the horizontal displacement $\Delta d$, observed in Figure 6a.

\section{Conclusions}

A novel analytical solution for the magnetic vector potential of an insulated coil immersed in a conductive medium has been derived under the simplifying assumption that the insulating dielectric enclosure surrounding the coil is infinitely wide. Numerical simulations show that this 
multilayer formula could be used to accurately estimate the mutual inductance of two coupled coils in a conductive medium over broad ranges of vertical and horizontal displacements between the coils and medium conductivity. The approximated expression of the mutual inductance has indeed been proved to exhibit very good accuracy, with an average relative error less than $2 \%$, provided that the radius of the insulating box is larger than three times the coil radius. Instead the previous solution for the field of a coil above a conductive slab [21] can be sufficiently accurate only when a medium with large conductivity is involved, e.g. $\sigma>40 \mathrm{~S} / \mathrm{m}$ for the coil configurations examined in this study. In particular, the multilayer solution is capable of correctly reproducing an interesting behaviour of the mutual inductance as a function of coil horizontal displacement, which exhibits oscillations with local minimum and maximum points without completely vanishing at some specific displacement as in free space coupling. This observation can be important in the research field of eddy current testing where the magnetic induction is utilized to detect or characterize some parameters of a conductive medium, e.g. metal detectors.

The proposed approach is versatile and can be easily adapted to various application scenarios, for instance, a submarine communications system consisting of two immersed coils close to the water surface. The sea may be considered as a conducting half-space and to derive a closed-form solution, an infinite layer of air should be added above the top of layer (I) and thus system (3) will contain 12 rather than 10 linear equations. Additionally, the approach is applicable when the dissipative layers have different conductivities, such as in free-floating wireless implantable neural recording [23] where a transmitting coil is placed on the scalp while the implantable neural recording sensors are placed on the cortex area of the patient brain under the skull. In this case, layer $(\mathbf{I})$ is air while layers (IV) and (VI), the skull and cortex respectively, have different conductivities.

Finally, our findings can have significant impact on the design and optimization of applications using MI waveguides embedded in conducting media (e.g., soil, sea water), body area networks, wearable and implanted sensors for biological systems, and wireless power transfer in RF-challenging environments.

This work was supported by EPSRC grant EP/N010493/1 as part of the SYMETA project (www.symeta.co.uk). The authors wish to thank fellow members of the Oxford Metamaterials Network (OxiMETA) for a number of stimulated discussions.

\section{References}

1. Z. Sun, I.F. Akyildiz, Magnetic induction communications for wireless underground sensor networks, IEEE Trans. Antennas. Propag. 58, 2426 (2010)
2. S. Kisseleff, et al., Channel capacity of magnetic induction based wireless underground sensor networks under practical constraints, in Proceedings of the Wireless Communications and Networking Conference (WCNC), 2013, pp. 2603-2608

3. M.C. Domingo, Magnetic induction for underwater wireless communication networks, IEEE Trans. Antennas. Propag. 60, 2929 (2012)

4. I.F. Akyildiz, E.P. Stuntebeck, Wireless underground sensor networks: research challenges, Ad Hoc Netw. 4, 669 (2006)

5. E. Shamonina, et al., Magneto-inductive waveguide, Electron. Lett. 38, 371 (2002)

6. E. Shamonina, et al., Magnetoinductive waves in one, two, and three dimensions, J. Appl. Phys. 92, 6252 (2002)

7. L. Solymar, E. Shamonina, Waves in metamaterials, (Oxford University Press, Oxford, 2009)

8. G.J. Palacky, Resistivity characteristics of geologic targets, in: M.N. Nabighian (Ed.), Electromagnetic Methods in Applied Geophysics, SEG, 1988

9. F. Ali, R. Sudhabindu, SAR analysis for handheld mobile phone using DICOM based voxel model, J. Microw. Optoelectron. Electromagn. Appl. 12, 363 (2013)

10. C.T. Tai, R.E. Robert, Radiation of a Hertzian dipole immersed in a dissipative medium, IEEE Trans. Antennas. Propag. 48, 1501 (2000)

11. J.R. Wait, The magnetic dipole antenna immersed in a conducting medium, Proc. IRE 40, 1244 (1952)

12. R. James, Insulated loop antenna immersed in a conducting medium, J. Res. Natl. Bur. Stand. 59, 02 (1957)

13. R. Moore, Effects of a surrounding conducting medium on antenna analysis, IEEE Trans. Antennas. Propag. 11, 216 (1963)

14. G.S. Smith, A theoretical and experimental study of the insulated loop antenna in a dissipative medium, Radio Sci. 8, 711 (1973)

15. R. King, Theory of the terminated insulated antenna in a conducting medium, IEEE Trans. Antennas. Propag. 12, 305 (1964)

16. R. King, et al., Insulated linear antenna: theory and experiment, J. Appl. Phys. 45, 1688 (1974)

17. S. Prasad, R. King, Experimental study of embedded insulated antennas, IEEE Trans. Antennas. Propag. 30, 1013 (1982)

18. R. King, et al., The electromagnetic field of an insulated antenna in a conducting or dielectric medium, IEEE Trans. Microw. Theory Tech. 31, 574 (1983)

19. A. Vallecchi et al., Impact of a conducting medium on the coupling of meta-atoms, in Proceedings of the 10th International Congress on Advanced Electromagnetic Materials in Microwaves and Optics (METAMATERIALS), 2016, pp. $385-387$.

20. A. Vallecchi, et al., Coupling between coils in the presence of conducting medium, IET Microw. Antennas. Prog. (submitted, under review)

21. C.V. Dodd, W.E. Deeds, Analytical solutions to eddy-current probe-coil problems, J. Appl. Phys. 39, 2829 (1968)

22. CST-Computer Simulation Technology, www.cst.com

23. P. Yeon, et al., Fabrication and microassembly of a mm-sized floating probe for a distributed wireless neural interface, Micromachines 7, 09 (2016) 


\section{Appendix}

The system of linear equations representing the boundary conditions is given by

$$
\begin{cases}A_{\mathbf{I}} e^{-\alpha_{1}(2 h)}-A_{\mathbf{I I}} e^{-\alpha(2 h)}-B_{\mathbf{I I}} e^{\alpha(2 h)} & =0 \\ A_{\mathbf{I}} \alpha_{1} e^{-\alpha_{1}(2 h)}-A_{\mathbf{I I}} \alpha e^{-\alpha(2 h)}+B_{\mathbf{I I}} \alpha e^{\alpha(2 h)} & =0 \\ A_{\mathbf{I I}} e^{-\alpha(h)}+B_{\mathbf{I I}} e^{\alpha(h)}-A_{\mathbf{I I I}} e^{-\alpha(h)}-B_{\mathbf{I I I}} e^{\alpha(h)} & =0 \\ A_{\mathbf{I I}} e^{-\alpha(h)}-B_{\mathbf{I I}} e^{\alpha(h)}-A_{\mathbf{I I I}} e^{-\alpha(h)}+B_{\mathbf{I I I}} e^{\alpha(h)} & =\mu_{0} I_{0} J_{1}\left(\alpha r_{0}\right) \\ A_{\mathbf{I I I}}+B_{\mathbf{I I I}}-A_{\mathbf{I V}}-B_{\mathbf{I V}} & =0 \\ A_{\mathbf{I I I}} \alpha-B_{\mathbf{I I I}} \alpha-A_{\mathbf{I V} \alpha_{1}+B_{\mathbf{I V}} \alpha_{1}} & =0 \\ A_{\mathbf{I V}} e^{\alpha_{1}(c)}+B_{\mathbf{I V}} e^{-\alpha_{1}(c)}-A_{\mathbf{V}} e^{\alpha(c)}-B_{\mathbf{V}} e^{-\alpha(c)} & =0 \\ A_{\mathbf{I V}} \alpha_{1} e^{\alpha_{1}(c)}-B_{\mathbf{I V}} \alpha_{1} e^{-\alpha_{1}(c)}-A_{\mathbf{V}} \alpha e^{\alpha(c)}+B_{\mathbf{V}} \alpha e^{-\alpha(c)} & =0 \\ A_{\mathbf{V}} e^{\alpha(2 h+c)}+B_{\mathbf{V}} e^{-\alpha(2 h+c)}-B_{\mathbf{V I}} e^{-\alpha_{1}(2 h+c)} & =0 \\ A_{\mathbf{V}} \alpha e^{\alpha(2 h+c)}-B_{\mathbf{V}} \alpha e^{-\alpha(2 h+c)}+B_{\mathbf{V I}} \alpha_{1} e^{-\alpha_{1}(2 h+c)} & =0\end{cases}
$$

where $\alpha_{2}=\alpha_{3}=\alpha_{5}=\alpha$ in free-space regions II, III and $\mathbf{V}, \alpha_{1}=\alpha_{4}=\alpha_{6}=\sqrt{\alpha^{2}+j \omega \mu_{0} \sigma}$ in conductive regions $\mathbf{I}, \mathbf{I V}$ and VI, and $\sigma$ is the conductivity of the medium. By solving (7), the unknown functions of the separation constant $\alpha$ which determine the magnetic vector potentials in the different layers are derived as in

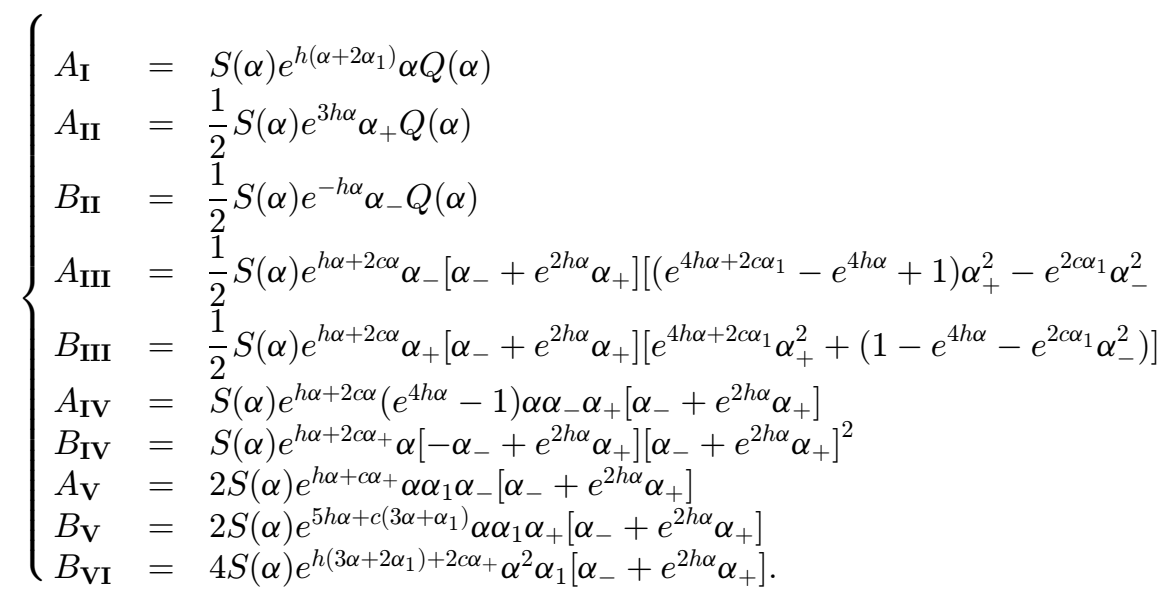

where

$$
\begin{cases}S(\alpha) & =\frac{\mu_{0} I r_{0} J_{1}\left(\alpha r_{0}\right)}{T(\alpha)} \\ T(\alpha) & =e^{2 c \alpha}\left\{e^{2 c \alpha_{1}}\left[\alpha_{-}^{4}+e^{8 h \alpha} \alpha_{+}^{4}\right]-\left(\alpha_{+} \alpha_{-}\right)^{2}\left[2 e^{4 h \alpha+2 c \alpha_{1}}+\left(e^{4 h \alpha}-1\right)^{2}\right]\right\} \\ Q(\alpha) & =e^{6 h \alpha+2 c \alpha_{+}} \alpha_{+}^{3}-e^{2 c \alpha_{+}} \alpha_{-}^{3}+e^{2 c \alpha}\left(e^{4 h \alpha+2 c \alpha_{1}}-e^{4 h \alpha}+1\right) \alpha_{+}^{2} \alpha_{-}-e^{2(h+c) \alpha}\left(e^{4 h \alpha}+e^{4 c \alpha_{1}}-1\right) \alpha_{+} \alpha_{-}^{2} \\ \alpha_{ \pm} & =\alpha \pm \alpha_{1}\end{cases}
$$

In principle, the analytical expressions of the magnetic vector potential in each region can be obtained by simply substituting the pertinent solution of system (7) into (3). In this paper our main interest is the induced voltage in the receiver and thereby the mutual inductance between two coils. Thus, only the expression of the magnetic vector potential in the receiver region - layer $(\mathbf{V})$ - is provided explicitly.

$$
\begin{aligned}
A_{\theta}^{(\mathbf{V})}(\rho, z) & =\int_{0}^{\infty}\left(A_{\mathbf{V I}} e^{-\alpha z}+B_{\mathbf{V I}} e^{\alpha z}\right) J_{1}(\alpha \rho) d \alpha \\
& =\int_{0}^{\infty}\left\{S(\alpha) J_{1}(\alpha \rho) 2 e^{\left(h \alpha+c \alpha+c \alpha_{1}\right)} \alpha \alpha_{1}\left[e^{\alpha z} \alpha_{-}+e^{-\alpha+2(c+2 h) \alpha} \alpha_{+}\right]\left[\alpha_{-}+e^{2 h \alpha} \alpha_{+}\right]\right\} d \alpha
\end{aligned}
$$

